Philosophie ANTIQUE

\section{Philosophie antique}

Problèmes, Renaissances, Usages

18 | 2018

L'athéisme antique

\title{
Le dieu de la loi
}

Athéisme et politique de la religion d'Euripide à Platon

\section{Fulcran Teisserenc}

\section{(2) OpenEdition}

\section{Journals}

Édition électronique

URL : https://journals.openedition.org/philosant/1008

DOI : 10.4000/philosant.1008

ISSN : 2648-2789

Éditeur

Éditions Vrin

\section{Édition imprimée}

Date de publication : 1 novembre 2018

Pagination : 37-69

ISBN : 978-2-7574-2372-1

ISSN : $1634-4561$

\section{Référence électronique}

Fulcran Teisserenc, "Le dieu de la loi », Philosophie antique [En ligne], 18 | 2018, mis en ligne le 01 novembre 2019, consulté le 02 décembre 2022. URL : http://journals.openedition.org/philosant/1008 ; DOI : https://doi.org/10.4000/philosant.1008

\section{(c) $)(9)$}

Creative Commons - Attribution - Pas d'Utilisation Commerciale - Pas de Modification 4.0 International - CC BY-NC-ND 4.0

https://creativecommons.org/licenses/by-nc-nd/4.0/ 


\title{
LE DIEU DE LA LOI \\ Athéisme et politique de la religion d'Euripide à Platon
}

\author{
Fulcran TeisserenC
}

Gramata, Université Paris 1

\begin{abstract}
RÉSUMÉ. À la fin du v viècle avant Jésus-Christ, apparaît dans la littérature grecque un certain nombre de discours impies. Platon en fait l'inventaire dans les Lois. Parmi les critiques adressées à la religion traditionnelle, l'une figure en bonne place chez Euripide : puisque les hommes injustes n'ont, dans les faits, guère à pâtir de leur conduite, il est légitime de mettre en doute l'existence des dieux. Or une autre thèse, voisine dans son vocabulaire mais conceptuellement distincte, se fait jour à la faveur de la première : l'irrespect de la loi et son affaiblissement entraînent une perte de confiance dans les dieux, car ces derniers sont une partie même du nomos. La réponse sophistique et même platonicienne à ce constat propose d'inverser les termes du problème : il faudrait pouvoir renforcer le tout par la partie, le nomos par la croyance aux dieux, les deux étant des faits institutionnels produits par le ou les législateurs. L'article examine comment cette suggestion se trouve développée, avec des variations, par Protagoras, Critias et l'Étranger d'Athènes au livre X des Lois.
\end{abstract}

Summary. At the end of the $5^{\text {th }}$ century B.C., several impious discourses appeared in Greek literature. Plato itemizes them in the Laws. Among the criticisms levelled at traditional religion, one is central to Euripides' writings: since, in practice, unjust men hardly ever suffer the consequences of their actions, the existence of the gods may be called into question. Another proposition, which hinges upon a similar lexical treatment even though it is conceptually different, stems from this observation: the disregard for and the undermining of the law (nomos) entail a loss of confidence in the gods, since the worship of the gods is regulated by the nomos. Sophistic thinkers and Plato alike address these views by looking at the problem from another angle: a bolstering of the parts (the articles of the law relating to the worship of the gods) would result in a strengthening of the whole (the nomos), as both are institutional facts which depend on the legislator(s) for their existence. This paper examines how this thesis is expounded by Protagoras, Critias, and the Athenian Stranger in Book X of the Laws.

Philosophie antique, ${ }^{\circ} 18$ (2018), 37-69 

La question de l'athéisme dans l'Antiquité occupe depuis peu une position moins marginale dans le paysage des recherches sur la pensée antique ${ }^{1}$. Pendant longtemps, la tendance voulait que le refus de toute espèce de dieu soit l'apanage des temps modernes, favorisé par les guerres de religion et l'émergence de la science expérimentale mais aussi par l'unité organique de dogme et de culte des christianismes, facilitant en quelque sorte le travail de négation. Avant le curé Meslier, mort en 1729, il n'y aurait pas eu d'athée déclaré. Le grand livre de Lucien Febvre sur le problème de l'incroyance au $\mathrm{XVI}^{\mathrm{c}}$ siècle s'était attaché à montrer que l'omniprésence de la religion chrétienne saturant le paysage et rythmant le temps pouvait s'accommoder d'un naturalisme qui recyclait les thèmes antiques de l'âme du monde ${ }^{2}$ et d'un humanisme imprégné de sagesse païenne, mais ne rendait pas possible la formulation explicite d'une vision du monde débarrassée de toute forme de surnaturel. Ce diagnostic méritait sans doute d'être nuancé, mais il a joué un rôle dans la perception de l'Antiquité, dont on ne présumait pas qu'elle eût pu être capable de s'émanciper de l'emprise religieuse. C'est pourtant ce que l'attention nouvellement portée à des œuvres ou à des textes, il est vrai marginaux ou minoritaires dans le corpus conservé de l'Antiquité, obligea à infirmer. Il devint possible de montrer sans trop de difficultés que non seulement il y eut des critiques de la religion traditionnelle, mais encore que des positions clairement athées furent défendues et argumentées. Ce qui ne laisse pas néanmoins de poser un problème de définition : qu'entendre par athéisme dans un contexte polythéiste ? La charge affective et sociale d'une telle position n'est évidemment pas de même nature qu'en contexte monothéiste, ou pour être exact (car le polythéisme n'exclut pas que se développe en son sein une forme de monothéisme), dans le cadre d'une religion universaliste à contenu révélé. Remettre en cause la nature des dieux du paganisme,

1. Voir, entre autres, Winiarczyk 1981 et 1984 ; Dorival et Pralon 2002 ; Sedley 2013 ; Whitmarsh 2015 ; Narbonne 2016.

2. Febvre 1942 p. 439-454; Foucault 1966 p. 32-59. 
nier leur existence, et nier l'existence de tout dieu, sont des démarches qui ont une portée très différente dans un univers religieux sans dogme arrêté, où la foi en tant que telle compte bien moins que la pratique et le rite ${ }^{3}$ et n'implique pas de dialogue personnel et intime avec le dieu ${ }^{4}$, où n'existent pas de révélation et d'écriture sainte, pas plus que n'existe un clergé chargé d'en préserver la lettre et l'esprit et de les déployer en dispositions légales, et où, enfin, l'opposition faux dieux/vrai dieu ne fait pas sens, pas plus que les phénomènes d'hérésie, en raison d'un principe de convertibilité du divin qui rend potentiellement traductible un panthéon dans un autre et interdit de dessiner une fois pour toutes ses limites 5 . Si donc le caractère polymorphe de la religion antique n'empêchait pas son analyse, y compris la plus radicale, il incitait plus à sa transformation ou à son usage intéressé qu'à son rejet pur et simple.

On peut distinguer très sommairement trois formes de déprise à l'égard de la croyance aux dieux dans l'Antiquité. La première, de nature logique, souligne les confusions d'un discours théologique : c'est ainsi que l'on trouve sous la plume de Sextus Empiricus une mise en lumière tant des incohérences internes du concept de divinité que de l'impossibilité de conclure de l'existence d'une idée à celle de son objet (comme le font pourtant toutes les théologies qui s'appuient sur la présence d'une prénotion de dieu dans l'esprit des hommes $)^{6}$. Certains cyniques pour leur part mettent le doigt sur la contradiction entre prescience divine et liberté humaine, injuriant au passage Apollon et ses prétendus oracles ${ }^{7}$.

La seconde espèce, à la fois ontologique et épistémologique, correspond aux courants matérialiste et sensualiste, dont l'athéisme, sans être proéminent et parfois même sans être confessé, semble pourtant logiquement impliqué. Figurent ici au premier chef les atomistes, Démocrite d'abord, Épicure et les épicuriens ensuite. À cette liste s'ajoutent ces personnages qu'évoque de manière énigmatique Platon sous l'appellation de « fils de la terre » (Sophiste, 246a-e), qui ne veulent reconnaitre comme être que ce qui est tangible, saisissable et attestable dans l'expérience sensible; ces « terriens » ont trouvé de dignes successeurs chez quelques cyrénaïques (Théodore l'Athée).

3. Voir Scheid 2005.

4. Voir Burkert 1985 p. 26.

5. Voir Assmann 2001 p. 82-95.

6. M. IX, 138-180 et 49, 2. Sextus rapporte aussi les arguments de Carnéade, les fameux sorites contre les dieux, qui ont pour office de montrer le caractère totalement indéterminé du divin, conduisant à y incorporer tout et son contraire. Voir Couloubaritsis 1989 et Couissin 1941.

7. Voir CEnomaüs de Gadara, Les Charlatans démasqués ou contre les oracles, fr. 5 (= Eusèbe, Préparation Évangélique, V, 21, 4). Voir Husson 2014.

8. Ici le dossier est considérable et controversé, non seulement quant à la doctrine exacte de chacun des auteurs, mais aussi quant à leurs relations mutuelles. Je ne l'ouvrirai pas. 
La troisième espèce de déprise est celle à laquelle va s'attacher ma présente réflexion. Elle est davantage politique et corrèle étroitement deux variétés d'existences au destin partagé, celle de la justice d'une part, celle des dieux d'autre part. Le constat de disparition de la première est en effet susceptible de provoquer un athéisme de protestation que l'on trouve formulé chez Euripide, lequel amorce également dans son œuvre une réflexion plus complexe sur l'intégration des dieux à la loi des hommes. Les modifications de cette dernière sont de nature à entraîner le déclin collectif des croyances religieuses qui en sont une composante. Mais c'est ouvrir du même coup la voie à des perspectives inverses d'un engendrement et d'un renforcement des dieux par le nomos, afin précisément de sauvegarder les conditions de sa propre mise en œuvre, voie dans laquelle se sont engagés tant certains sophistes (Protagoras et Critias) que Platon lui-même.

\section{Euripide ou l'injustice en balance}

La crise religieuse dont témoignent certains personnages d'Euripide n'est pas liée à l'existence du mal comme tel, mais à celle du mal impuni. Pourquoi faire encore crédit à la tradition et aux croyances reçues des ancêtres quand on observe combien la vie réelle des hommes s'écarte des régularités dont les dieux sont supposés être les garants ? La question soulevée par l'existence d'une injustice victorieuse n'est pas d'abord celle de la théodicée, c'est-àdire celle de la compatibilité du mal et d'un principe divin organisateur du monde. La préoccupation que fait exprimer Euripide par ses personnages est plus simple : ils s'alarment d'une faute échappant à la sanction qu'elle appelle pourtant. De même, le juste frappé par le sort, ou victime de la malignité d'autrui, n'est pas en lui-même source d'étonnement ; c'est bien plutôt qu'il ne soit pas mis un terme à ses épreuves et que sa valeur reste ignorée et bafouée. Le déficit de justice qui heurte la sensibilité des personnages d'Euripide est moins le déficit de la justice préventive que le déficit de la justice réparatrice - de cette justice corrective et réactive destinée à rétablir le droit et à compenser le préjudice, qui suppose comme préalable l'existence même du mal.

De ce point de vue, le mode d'action et de gouvernement des dieux devrait pouvoir se lire dans le bonheur des justes et le malheur des méchants. Si tel n'est pas le cas, alors il faut bien admettre qu'ils ont déserté le monde. Cette argumentation est très largement répandue dans l'œuvre d'Euripide et elle correspond à un certain sentiment qui devait être assez partagé à l'époque.

Ainsi Électre : « il faudrait ne plus croire aux dieux, si l'injustice l'emporte » (Électre, 583), ou la mère d'Iphigénie : « s'il y a des dieux, le juste que tu es les trouvera équitables ; s'il n'y en a pas, nos efforts actuels ne servent à rien » (Iphigénie en Aulide, 1034-1035). Il n'y a plus d'alternative dans les propos de l'impie Bellérophon : 
Il n'y a pas de dieux, quoi qu'on raconte, car des tyrans cruels et impies sont souvent plus heureux que des hommes ou des cités qui honorent les dieux. (Fr. 286 Nauck 1912)

Qu'il s'agisse d'une inquiétude répandue à Athènes, la preuve en est donnée par le livre $\mathrm{X}$ des Lois, qui dresse un portrait des trois sortes d'impies (le négateur des dieux, le partisan de leur indifférence, celui de leur corruptibilité) que Platon observe chez ses contemporains. Le profil de Bellérophon correspond assez exactement à ce que le philosophe dit de la première espèce, qui constaterait dans le monde une injustice trop caractérisée pour devoir admettre la réalité d'un gouvernement divin.

Le Cyclope en offre une autre variante, illustrant cette tyrannie triomphante dont Bellérophon reconnaissait la regrettable prévalence. Poursuivant ici les indications laissées par Homère ${ }^{9}$, Euripide en fait, dans la pièce portant son nom, un personnage emblématique d'un matérialisme grossier et primitif, refusant les artifices de la civilisation, aussi bien les lois de l'hospitalité que celles du culte. Son impiété est subversive. Il ne se connaît pas de maître, raison pour laquelle il se dit dieu et s'irrite de se voir inciter par Ulysse à la piété, notamment à l'égard de son père Poséidon. Pas davantage ne redoute-t-il la foudre de Zeus (qui «n'est pas un dieu plus puissant que moi : au surplus, je ne m'en soucie guère »). Seul compte son ventre («le plus grand des dieux $\gg, 335$ ), centre de ses plaisirs et de son énergie sexuelle, dont le pauvre Silène risque de faire les frais. C'est donc lui-même que le Cyclope entend honorer : « Je me garde bien de les immoler (les troupeaux) à quelque autre dieu qu'à moi-même » (334). L'《 autel »du sacrifice est la cuisine de la caverne où il va découper et cuire les compagnons d'Ulysse, victimes nécessaires à son appétit cannibale, dans une parodie manifeste des rituels qu'il exècre (346).

À la divinisation de son corps, le Cyclope ajoute celle d'une activité (« est Zeus, pour les hommes sensés, le manger, le boire et l'absence de soucis »), qui culmine dans l'apothéose de « la richesse » (《le dieu des sages », 316) qui rend possibles les jouissances sensibles et l'insouciance. La vie apolitique du Cyclope conjugue donc la négation des dieux traditionnels et le mépris de la loi, au profit de sa seule force vitale et des plaisirs qui l'accompagnent.

D'autres personnages d'Euripide sont moins radicaux, mais évoquent également, par contraste avec l'ordre attendu d'une nature et d'une polis accueillantes aux dieux, l'existence d'un monde livré aux puissances du hasard, à la tuche. Ainsi, dans Hécube, le héraut Thaltibios s'exclame :

Que penser, ô Zeus ? Veilles-tu sur les hommes, ou est-ce en vain que tu as cette réputation? Est-ce faux, ce qu'on croit, qu'il existe des dieux ? Le hasard

9. Pour une différence notable cependant, relativement aux questions débattues dans cet article, voir la note 20 . 


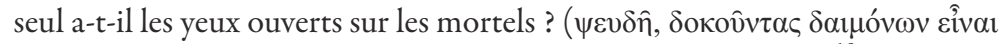

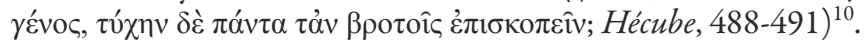

Dans le Cyclope, Ulysse invoque à son tour Héphaïstos et Hypnos : « n'abandonnez pas Ulysse, sinon il faut tenir le hasard pour un être divin et les divinités pour inférieures au hasard $\gg(606)$. Au vers 355, il déclarait carrément :

Zeus hospitalier, vois où j'en suis ; si tu ne regardes pas, c'est en vain que Zeus est honoré, car il est un dieu qui compte pour rien (ă $\lambda \lambda \omega \varsigma$ vo

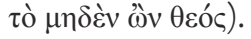

Le chœur d'Hippolyte chante :

Combien les soins qu'ont les dieux pour les hommes, lorsque j'y pense, me relèvent de ma peine! Mais, tout en espérant, au fond de moi-même, comprendre, je cesse de comprendre lorsque mes yeux s'ouvrent aux actions des mortels et à leurs sorts. Car il en sort tantôt ceci, tantôt cela, et toujours la vie humaine est instable et va de-ci, de-là. (1104-1110)

À ce hasard caractéristique de la vie humaine, ballottée en tous sens, sans qu'aucun ordre puisse s'en dégager, il faudrait ajouter l'incohérence, la versatilité et la mobilité des desseins prêtés aux dieux, qui font précisément douter du divin en lui-même.

Quel mortel, après de profondes recherches, dira ce qui est divin, ce qui ne l'est pas, ou ce qui est d'une nature intermédiaire ? Quel accomplissement considérable, pour celui qui discerne ce qui relève du divin, ici et à nouveau là-bas, chargé d'un sens contradictoire, surgissant au gré des événements les plus inattendus! (Électre, 1138-1143)

À nouveau, le vocabulaire et la thèse rappellent exactement les cibles de Platon au livre $\mathrm{X}$ des Lois, dont le propos est de démontrer contre les athées que l'art est premier par rapport à une phusis aveugle et à la tuche (888e6-889c6).

Soutenir l'ordre inverse, c'est, par un phénomène de correspondance entre le discours et son objet, faire un usage hasardeux du logos, si l'on en croit les déclarations du chœur s'en prenant dans une pièce non identifiée à

celui qui, contemplant cela, n'enseigne pas tout d'abord que son âme considère un dieu, et ne rejette pas très loin de lui les opinions trompeuses de ces gens qui bavardent à propos du ciel, dépourvus de toute intelligence, et dont la langue insensée tire au hasard des traits sur ce qui demeure caché. (Fr. 913, Kannicht 2004)

Là encore, le sentiment ici exprimé est tout à fait comparable à celui que

10. Un fragment du Phrixus B (fr. 820 Kannicht 2004) dénonce les « mortelles divagations » des hommes, affirmant que « le hasard existe, mais non les dieux », tout en retenant l'alternative : « si le hasard existe, il n'est pas besoin des dieux, et si les dieux sont forts, le hasard n'est rien ». 
l'on trouve dans la bouche de Clinias dans les Lois, quand il condamne ces impies qui, sous l'inspiration d'Anaxagore, identifient les astres à leurs matériaux (X, 886a2-4, cf. 886d5-e2).

Mais, surtout, s'amorce, chez Euripide, une autre manière de remettre en cause la continuité des ordres politique et cosmique. La défiance à l'égard d'une loi humaine conduirait naturellement à affaiblir cela même que cette loi prescrit, qui est tout autant de l'ordre du faire que du croire. En témoigne par exemple la requête d'Hécube à Agamemnon, qui exprime son amour inquiet pour la loi des hommes, laquelle commande la confiance qu'ellemême (Hécube) est prête à accorder aux dieux :

Oui, je suis esclave et faible sans doute, mais les dieux sont puissants. Puissante aussi celle qui les gouverne, la Loi. C'est parce qu'elle existe que nous croyons qu'il est des dieux, et que nous réglons notre vie

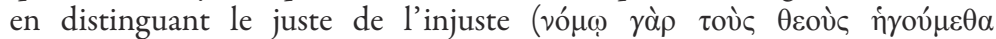

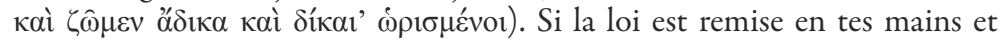
s'y trouve ruinée, s'il n'y a point de châtiments pour ceux qui tuent leurs hôtes ou qui osent piller la demeure des dieux, l'équité disparaît de la vie des hommes. (Hécube, 798-805)

Ce texte fait glisser le nomos d'un sens à un autre ; il peut d'abord être entendu comme nécessité impersonnelle, loi cosmique s'imposant à tous, y compris les dieux ${ }^{11}$. Mais, dans la deuxième phrase, une ambiguïté apparaitt ; on pourrait à la rigueur comprendre que c'est la manifestation de cette loi, sa réalisation concrète, qui fait croire aux dieux, en prêtant alors à vó $\mu \omega$ la valeur existentielle d'un effet qui inciterait à remonter à sa cause extérieure - de re (sans que celle-ci soit donc contenue - obliquement - dans l'effet, comme le serait en revanche une proposition définissant le contenu de dicto d'une croyance ou d'un acte législatif). Mais il est possible de comprendre autrement : la croyance aux dieux est un article de la loi, elle fait donc partie des traditions et des institutions. Les dieux seraient donc inclus dans le cosmos humain en tant qu'il est façonné par la loi au sens large. Or la troisième phrase favorise cette interprétation, dans la mesure où il est dit que la loi est remise entre les mains du prince : il ne peut s'agir que d'une loi humaine; quand les hommes ne la suivent plus, alors « l'équité disparaît » certes, mais aussi la croyance aux dieux - et les dieux eux-mêmes, par conséquent.

Dans Les Troyennes, Hécube formule explicitement l'alternative que nous venons de dégager - qui restait encore sous-jacente dans les propos qu'elle tenait dans la pièce portant son nom : à présent (vers 886), elle pose directement la question de la véritable nature de Zeus :

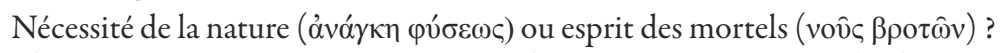

Les deux options envisagées correspondent très exactement à la distinc-

11. Voir Alceste : « je n’ai rien trouvé de plus fort que la nécessité » dit le chœur (965). Voir aussi Hélène, 513 sq. et les frag. 330, 415, 757 Nauck 1912. 
tion entre une loi anonyme et souveraine et un ensemble de croyances dont la raison d'être serait sociale et psychologique : la dernière désignation (« esprit des mortels ») introduirait en effet la possibilité que ce nom de Zeus ne renvoie qu'à une idée dans l'esprit des hommes, qu'à une certaine représentation plus ou moins collective. Le même personnage dit à Hélène, un peu plus bas dans la pièce, que son esprit (vô̂ৎ) s'est changé en Aphrodite (988) : la déesse n'est qu'une projection, une hypostase, des inclinations érotiques

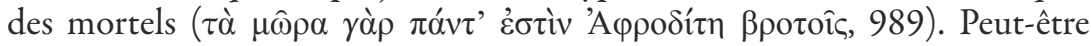
aussi faut-il admettre la même origine pour Zeus, comme le laisse entendre ce fragment célèbre, selon lequel «l'esprit en chacun de nous est un dieu » (frag. 1018 Nauck). En tout cas, le début de Mélanippe la sage, du moins la première version, corrigée ensuite en raison de l'émotion du public ${ }^{12}$, offre à cet égard un éclaircissement sans équivoque à propos du dieu suprême :

De Zeus, quel qu'il puisse être, je ne sais rien, sinon les histoires contées à son sujet.

De sorte que l'on passe d'une forme d'athéisme à une autre : d'une remise en cause des dieux incapables de faire régner la justice à une remise en cause de la loi incapable d'établir cet ordre dont les dieux sont parties prenantes. La loi vacillant, ce sont aussi les dieux auxquels elle enjoint de croire qui disparaissent, non pas de la nature en elle-même, mais du monde que la loi édifie. De ce point de vue, la loi qui « gouverne les dieux »n'est plus une nécessité impersonnelle et cosmique, elle s'identifie à l'ensemble de ces traditions, coutumes et croyances qui se rapportent à eux, qui les font exister d'une certaine manière. Et n'est-ce pas, en toute rigueur, à cet ensemble qu'est imputable l'enseignement de « la différence du juste et de l'injuste » ?

La distance est d'ailleurs courte entre la reconnaissance de l'ancrage anthropologique et social de la croyance aux dieux et la piété conservatrice. Quand Tirésias s'oppose dans les Bacchantes aux nouvelles conceptions des sophistes, il revendique le legs des anciens, l'héritage de la tradition, les coutumes des ancêtres, « aussi vieilles que le temps ».

Inutile de sophistiquer avec les divinités. Les traditions de nos pères, [...] nul raisonnement ne les jettera bas, quelque subtilité que découvrent les esprits les plus profonds (200-204).

Il s'agit ici de l'empire de ce qui s'est toujours fait et s'est toujours cru. Par cette évocation, Tirésias ne prétend pas fournir des indices ou des arguments destinés à établir la validité objective de la croyance, mais il veut souligner le rattachement de sa propre conduite et de son attitude face aux dieux à la permanence de la communauté et à son monde.

Une même fidélité à la puissance du nomos, et à ses dieux, s'exprime dans

12. L'anecdote ainsi que les deux versions du vers sont rapportées par Plutarque (Amatorius, 756b). 
un couplet remarquable du chœur. S'attaquant à ceux qui

honorent ce qui est insensé et ne grandissent guère les dieux, à ceux qui masquent de multiples manières la longue cadence du temps et poursuivent les choses profanes,

(un portrait dans lequel on reconnaîtra aisément l'athée, que visait à l'instant la critique de Tirésias), le chœur déclare qu'il faut pourtant considérer qu'il n'y a

rien de plus fort que les lois, et qu'il ne coûte guère de croire à ce principe puissant, quelle que soit la nature du divin, en cet usage (vó $\mu \mu$ ov) que le

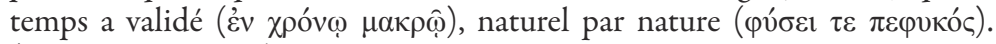
(Bacchantes, 892 sq.)

Voilà réunis les termes de vó $\mu$ oc et de $\varphi v ́ \sigma ı \varsigma^{13}$, par l'intermédiaire du

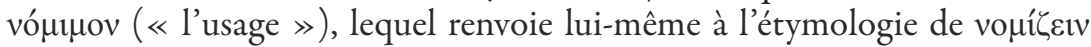
(« croire ») $)^{14}$. Or la loi immémoriale prolonge la nature non seulement parce qu'elle bénéficie d'une forme de permanence et de stabilité, mais encore parce qu'elle procède « naturellement » de la nature, à suivre les propos de Tirésias lui-même. Confronté à la nouveauté apparente du dieu Dionysos, et à la prétention de ce dernier à être reconnu et à faire l'objet d'un culte public au même titre que les autres dieux, il propose de l'insérer dans la religion traditionnelle en lui aménageant une fonction et une origine qui font pendant à celles de Déméter.

Il y a deux divinités, ô jeune homme, qui tiennent le premier rang chez les hommes. L'une est la déesse Déméter, ou la Terre, donne-lui le nom que tu voudras ; c'est elle qui d'aliments solides nourrit les mortels. L'autre s'est placée de pair avec elle : c'est le fils de Sémélé ; il a trouvé un breuvage, le jus de la grappe, et l'a introduit parmi les mortels pour délivrer les malheureux hommes de leurs chagrins en les abreuvant de la liqueur de la vigne. Le sommeil, l'oubli de leurs maux quotidiens, voilà son présent; il n'y a pas d'autre remède à leurs peines. Lui, qui est un dieu, s'offre en libation aux dieux : c'est donc à lui que les hommes doivent leurs biens. (274-285)

La théorie que formule ce passage reprend l'opposition classique depuis la philosophie ionienne entre le sec et l'humide, constitutifs du corps du monde et de l'homme. Elle identifie Déméter et Dionysos à leurs formes les plus éminentes, le pain et le vin respectivement. Et elle s'inspire manifestement de Prodicos qui attribue l'origine des dieux à la déification des choses utiles à

13. Cf. Ion, 642. Certains sophistes opposaient l'un à l'autre : Hippias, 86DK C1, Archélaos, 60DK A1, Antiphon, 87DK B44, et, bien sûr, le personnage de Calliclès dans le Gorgias.

14. Nomizein theous : « le fait de reconnaitre les dieux en leur accordant le culte prescrit par le nomos ("la loi, la coutume ") » (Burnet 1924, p. 104), reconnaissance qui n’est guère éloignée de celle par laquelle, dans les pactes d'alliance, les deux cités liées s'engagent à « considérer (nomizein) comme amis et ennemis les mêmes peuples » (voir Fahr 1969). 
l'homme et à celle de leurs inventeurs ${ }^{15}$. Le passage s'effectue aisément, dans la bouche de Tirésias, de l'élément au dieu (de la terre à Déméter), et inversement, du dieu à la chose qu' il symbolise (« lui qui est un dieu, il s'offre en

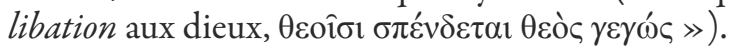

Qui plus est, Tirésias adopte une lecture « rationaliste » des mythes : il explique à Penthée qu'il n'est pas nécessaire de croire littéralement que Dionysos enfant aurait été cousu à l'intérieur de la cuisse de Zeus. Cette histoire serait née d'une confusion sur les mots (286-289), découlant d'une tromperie : Héra aurait été abusée par un morceau d'éther façonné à la ressemblance de Dionysos ${ }^{16}$. C'est encore, même par équivoque, rattacher le nouveau dieu à un élément naturel ${ }^{17}$.

Pour autant, il n'y a pas lieu de faire de Tirésias le partisan d'un athéisme strict : ce serait contradictoire avec ses autres déclarations - notamment celle selon laquelle, possédé par le dieu, le devin a une prescience de l'avenir (298-301), et celle que nous avons citée, exprimant son attachement au cosmos éthique légué par ses ancêtres et le refus de toute sophistication. Reste que sa conception de divinités enracinées dans la générosité de la nature - s'accordant avec les nombreux rites de fertilité et faisant écho à la représentation largement répandue d'une agriculture providence ${ }^{18}-$, rejoint la thèse imputant la formation des religions à la tendance de l'esprit humain à témoigner reconnaissance et gratitude à des êtres spontanément placés à l'origine des bienfaits qu' il expérimente dans sa vie mortelle.

Il serait bien imprudent de conclure des personnages à l'auteur, d'attribuer directement à Euripide, comme exprimant sa véritable pensée, les propos impies ou athées que tiennent Hécube ou Bellérophon. Les Troyennes enseignent au spectateur que les dieux n'ont pas oublié Troie et préparent, à l'insu même d'Hécube, la vengeance que celle-ci désire. Les fragments conservés de la tragédie perdue Bellérophon permettent de penser que le héros éponyme est finalement châtié, foudroyé par Zeus ${ }^{19}$. Le Cyclope est aveuglé $^{20}$. La pièce Les Bacchantes montre un Dionysos qui n'attend pas

15. 84DK B5 : « [...] ce qui a été écrit par <Prodicos> sur le fait que les denrées et les ressources ont <les premières> été considérées comme des dieux et honorées, puis ceux qui avaient découvert les moyens de se nourrir ou de se protéger ou les autres arts, comme Déméter, Dionysos ... »(Philodème, Sur la piété, col. IX, 7). Témoignages en même sens de Minucius Felix et de Cicéron ; celui de Sextus se contente pour sa part de mentionner la déification des choses.

16. La méprise d'Héra est symétrique de celle de Penthée : elle ne voit pas l'éther dans Dionysos, il ne voit pas Dionysos dans l’Étranger.

17. À maintes reprises, Euripide suggère aussi l'éventualité que Zeus soit un principe physique comme l'éther. Voir fr. 877. Cf. fr. 839, 919, 941 Nauck.

18. Thémistius à propos de Prodicos (Discours 30, 422 Dindorf $1832=84 \mathrm{DK}$ B5).

19. Voir Riedweg 1990 p. 52.

20. Dans l'Odyssée, le châtiment infligé par Ulysse est compris par ce dernier comme la réplique des dieux à l'impiété du Cyclope (479). Mais ce motif est absent de la pièce d'Euripide. 
spécialement d'être honoré comme un bienfaiteur de l'humanité, contrairement à ce que souhaitent Tirésias et Cadmos, pas plus qu'il ne désire être compris et interprété à la lumière d'une « humaine sagesse » (395-396). Il veut être reconnu pour ce qu'il est et force les Thébains à élargir leur expérience du divin ${ }^{21}$.

Le contexte compte, à l'évidence. M. Lefkowitz insiste sur le fait que les dénouements ne sont jamais favorables aux impies dans les pièces d'Euripide $^{22}$. Mais une conclusion édifiante pouvait précisément offrir au développement qui précède l'occasion de faire le procès des dieux et de mettre en avant un athéisme bravache, que l'on pouvait d'autant mieux exposer et rendre persuasif qu' il serait de toute façon puni au terme de la narration ${ }^{23}$. Et le public, tout comme Euripide, n'ignorait pas la différence entre la fiction et la réalité. Le réconfort d'un châtiment ex machina de l'impudent ne devait certainement pas suffire à lever les doutes.

De surcroît, les dieux, chez Euripide comme d'ailleurs chez Sophocle ${ }^{24}$, sont avant tout désireux d'être le plus largement honorés et se montrent très sourcilleux quant au respect de leurs prérogatives. Pour le reste, ils sont plutôt indifférents à la souffrance des hommes. Ils aiment à voir leur propre cause habilement et bravement défendue par les mortels qu'ils ont distingués. Cette « psychologie » divine les expose, il est vrai, à une nouvelle contestation ironique ${ }^{25}$. Car admettre qu'ils sont animés par des motifs de cet ordre revient, volens nolens, à les mettre dans la dépendance des hommes. Un dieu en quête de reconnaissance est un dieu dont l'existence est fragilisée, à la merci du culte et de ses fluctuations, c'est un dieu éminemment mortel, comme le montrera de façon caustique Lucien de Samosate dans Zeus tragédien.

La conduite incohérente et injuste des dieux peut bien susciter une remise en cause de leur divinité dans la créance de certains des personnages d'Euripide ( « si les dieux font quelque chose de honteux, ce ne sont pas des

21. Voir Vernant 1985 p. 31-58.

22. Lefkowitz 2003 p. 105.

23. Voir Whitmarsh 2014 p. 113-114. Ajoutons Molière : Don Juan est puni sans doute, mais le mal est fait, l'indifférence libertine à Dieu est une possibilité intellectuelle, morale et existentielle qui a acquis sa légitimité propre.

24. Chez Sophocle, pas de mise en cause de l'existence des dieux, bien qu' ils soient déconnectés de toute justice. Voir par exemple Philoctète, 446-450. Antigone ne menace pas Créon de la vengeance des dieux, comme s'ils pouvaient être les agents d'une justice supérieure. Chez OEdipe et Ajax, le malheur n'est pas le fruit d'une justice divine compréhensible, ni même d'une justice tout court : Sophocle constate plutôt « l'immense indifférence des dieux », « l'œuvre funeste de Zeus » (Trachiniennes, 1266 jusqu'à la fin). Pour l'ensemble de la question, voir Dodds 2009, p. 115-116.

25. Voir les propos prêtés à Zeus dans le Banquet (190d3) et, déjà, ceux d'Hermès dans l'Odyssée (IV, 100-103). 
dieux $\gg^{26}$ ), un fond divin subsiste assurément chez lui, à tout le moins dans un écart avec les Olympiens et leur épiphanie, dans les marges de la religion civique. Quelque chose de sacré irradie dans l'enveloppe cosmique de l'Éther. Mais surtout, en contraste avec ce principe lumineux, des forces obscures se dérobent au regard et leurs manifestations contradictoires et soudaines désarçonnent et aveuglent les hommes. Il demeure toutefois qu'une figure traditionnelle des dieux est ébranlée par le désordre du monde ${ }^{27}$. Qu'elle s'efface, ainsi que la loi qui la règle, et que vienne à surgir un visage plus inquiétant et déroutant du divin ${ }^{28}$, c'est un des ressorts du drame euripidien. De ce fait, il intègre nécessairement une position critique à l'égard de la religion de la cité. Or c'est ce moment « athée » que nous observons chez d'autres esprits contemporains d'Euripide, avec lesquels probablement il partageait certaines composantes d'une vision moins naïve du fait religieux.

\section{Perspectives sophistiques sur la valeur et l'usage des croyances religieuses}

Aristophane, qui a beaucoup contribué à la réputation d'athéisme qui frappa Euripide, fait largement état à son tour des éléments d'une critique des dieux. Son intention est parodique et bouffonne ; le poète comique ne partage assurément pas le point de vue qu'il rapporte. Mais la caricature préserve, voire concentre, la force subversive du propos. Outre la plainte de cette commerçante de couronnes religieuses qui reprochait à Euripide de lui avoir fait perdre la moitié de son chiffre d'affaires par sa propagande contre les dieux (Thesmophories, 450-451), outre les analogies entre phénomènes célestes et phénomènes culinaires et gastriques ${ }^{29}$ par lesquelles est suggérée la présence de mécanismes naturels derrière les météores, Aristophane présente un Socrate doutant que les dieux fassent respecter, par leur intervention dans le monde des hommes, une quelconque justice. Le fonctionnement aveugle de la nature ne révèle en effet aucune providence.

Strepsiade. Mais la foudre, d'où lui vient son étincelle de feu, dis-le-moi, qui tantôt nous frappe et nous consume, tantôt laisse vivants ceux qu'elle a effleurés ? Il est évident que c'est Zeus qui la lance sur les parjures.

Socrate. Mais comment, sot que tu es, toi qui sens l'âge de Cronos, plus vieux que le pain et la lune, s'il frappait les parjures, comment n'aurait-il pas fou-

26. Bellérophon, fr. 292 Nauck.

27. Pour une lecture dialectique du divin chez Euripide, qui évite de trop durcir l'altérité de Dionysos, voir Blaise 2003.

28. Ainsi, dans Hippolyte, « Kypris apparemment n'est pas une déesse, mais quelque chose de plus grand » (359-360). Plus haut, la nourrice déclarait : « Toute la vie des hommes est pleine de douleur, et il n'est point de relâche à leurs maux ; mais s'il est quelque chose d'autre, de plus précieux que la vie, les ténèbres l'enveloppent et nous le cachent » (189-192).

29. Également présentes dans le Cyclope (325-330). 
droyé Simon, Cléonyme, Théoros ? Ce sont pourtant bien des parjures. Mais il frappe ses propres temples et Sounion, le cap de l'Attique, et les grands chênes. À moins que le chêne ne soit parjure ? (Nuées, 394-403)

Or Diagoras, que l'Antiquité s'accordait à considérer comme un athée, et auquel font justement allusion les Nuées (830) sous la désignation transparente de Socrate le Mélien ${ }^{30}$, a lui-même largement développé cet argument, à suivre du moins le témoignage de Cicéron (De natura deorum, III, 37$)^{31}$.

Étant à Samothrace, on montra à Diagoras des ex-voto de personnes réchappées d'un naufrage. « Regardez cela, lui dit-on, vous ne croyez pas qu'il y ait une providence en laquelle il faut croire ? » Ce à quoi Diagoras répondit ceci : «Je ne m'étonne pas de voir les tableaux de ceux qui sont réchappés. La coutume est que l'on peigne ces gens-là. Mais on ne s'avise nulle part de représenter ceux qui périssent sur mer tout en ayant cru à la même providence. »

L'idée de providence induit un biais cognitif ; ces événements qui arrivent sans que les hommes en saisissent les causes sont interprétés en fonction du sens accidentel qu'ils revêtent pour eux ; s'ils sont heureux, c'est qu'ils correspondent à une gratification divine, s'ils sont malheureux, c'est à titre de conséquence d'un courroux divin. Or cette conviction ne se maintient qu'à condition d'écarter résolument de la vue de l'esprit tout ce que produisent par ailleurs ces phénomènes censément manipulés par les dieux : la destruction du temple de Zeus frappé par la foudre, la noyade sans trace de l'homme pieux dont le navire est coulé par la tempête.

Autre anecdote de même portée, rapportée par Cicéron :

Diagoras à bord d'un vaisseau traversait une forte tempête en mer. Pendant le gros temps, on se mit à dire à Diagoras que l'équipage et les passagers méritaient ce qu'ils enduraient puisque le bateau transportait un impie. Ce à quoi Diagoras répondit : «Regardez les autres navires en ce moment dans la même tempête que nous. Croyez-vous que je suis aussi sur chacun de ces bâtiments ? » (De natura deorum, III, 37).

Les crédules imaginent donc que le bateau serait attaqué par la tempête en raison de l'impiété d'un des passagers ${ }^{32}$ : mais les autres bateaux sont-ils

30. Voir le scholiaste et Winiarczyk 1981, T6A2. Voir aussi Oiseaux, 1072-1073 (« En ce jour plus que jamais on proclame : celui de vous qui tuera Diagoras le Mélien recevra un talent, celui qui tuera l'un des tyrans morts recevra un talent »), et Grenouilles, 320.

31. Whitmarsh 2016 propose d'interpréter le titre énigmatique de l'ouvrage de Diagoras 'A $\pi$ лор de siège intellectuel ». Ferait allusion à ce titre le dernier vers du fragment 286 du Bellérophon, qui, après une lacune, évoque « les terribles désastres qui fortifient ( $\pi$ vyov̂ $\left.\sigma^{\prime}\right)$ les choses divines ». C'est cette construction religieuse que Diagoras dans son œuvre se serait efforcé de démolir en montant, comme Bellérophon, à l'assaut de l'Olympe.

32. Euripide fait état de la croyance selon laquelle les dieux font couler un bateau et périr les voyageurs innocents pour perdre un impie qui était à bord (Électre, 1355). Voir aussi Ésope, 
épargnés ? Cet argument file la métaphore maritime précédente. Mais on ne peut oublier que le navire est aussi l'emblème de la cité3 ${ }^{33}$. Or on a cru pendant longtemps que «souvent toute une ville a porté la peine des crimes d'un homme » (Les Travaux et les Jours, 240) ${ }^{34}$. L'anecdote de Diagoras remet donc en cause cette idée d'une sanction divine collective (dont les manifestations concrètes suggérées frappent en réalité indistinctement le pieux et l'impie, la cité pieuse et la cité impie) ; non seulement l'innocence ne protège pas de la tempête (les bateaux menacés ne transportent pas tous des Diagoras), mais encore l'impiété ne déclenche pas le naufrage (le bateau qui convoyait Diagoras est finalement arrivé à bon port). Application politique : ni la présence d'Anaxagore n'a provoqué la ruine d'Athènes, ni l'innocence des Méliens ne leur a épargné la cruauté des Athéniens.

On n'en sait guère plus à propos de Diagoras. Peut-être n'était-il pas un athée au sens strict, niant l'existence d'êtres plus parfaits que les hommes, et se contentait-il de mettre en lumière les distorsions causales que présentent leurs actions. En revanche, un autre contemporain d'Euripide, dont on dit qu'il était l'ami, semble avoir déployé de façon plus substantielle une partie de cet athéisme dont on trouve des traces éparses dans l'œuvre du plus jeune des trois grands poètes tragiques. Du moins disposons-nous à son sujet de témoignages un peu plus détaillés, qui permettent d'unifier davantage la constellation intellectuelle que nous esquissons.

Rappelons d'abord que les Bacchantes contiennent une allusion assez claire à l'athéisme, ou la doctrine réputée telle, de Protagoras. En effet, Tirésias, dont nous avons déjà parlé, déclare, on s'en souvient :

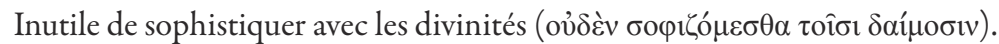
Les traditions de nos pères [...], nul raisonnement ne les jettera bas ( $\kappa \alpha \tau \beta \beta \alpha \lambda \varepsilon \hat{)}$ ), quelque subtilité que découvrent les intelligences les plus pro-

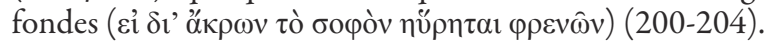

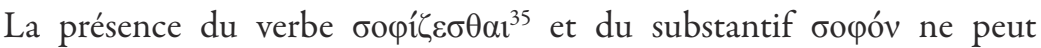
manquer de faire penser en effet au plus éminent des sophistes, celui dont

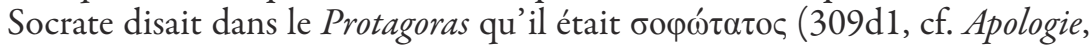
20a3). En outre, le verbe $\kappa \alpha \tau \alpha \beta \alpha \lambda \varepsilon \hat{\imath}$ paraît être une allusion transparente au titre de son célèbre ouvrage, Les Arguments Renversants (ou Terrassants),

Fable 48, L'homme mordu par une fourmi et Hermès, à propos de la punition de tout un navire par les dieux pour châtier un impudent.

33. Voir, par exemple, l'association de la cité et du navire, avec la métaphore du naufrage découlant de «l'ignorance la plus grave » de ses membres, dans Platon, Politique, 302a (voir aussi Plt. 299a, Clit. 408b, Grg. 511e, R. 488b-e).

34. La maxime d'Hésiode était encore dans la bouche d'Eschine un argument redoutable dans sa lutte contre Démosthène (Sur la fausse ambassade, 158, 7). Voir n. 67 l'usage qu'en fait Platon.

35. Dans le Phèdre (229c7), Socrate utilise le même verbe pour décrire l'attitude de ceux qui rationalisent les mythes. 


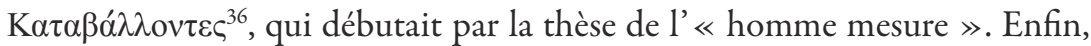
le contenu même du reproche formulé par Tirésias - de mettre en cause la religion traditionnelle - correspond exactement à ce que fit Protagoras, dans l'autre thèse pour laquelle il est connu, thèse qui défraya la chronique et qui, au dire de certains, lui valut un procès un impiété :

Des dieux, je ne puis savoir ni qu'ils existent, ni qu'ils n'existent pas, ni quelle est leur forme : car beaucoup d'obstacles m'empêchent de le savoir, l'obscurité et la brièveté de la vie de l'homme ${ }^{37}$.

Parmi les raisons de ce qui se présente à première vue comme un agnosticisme, l'« obscurité » découle, semble-t-il, du fait que les dieux ne sont pas un objet d'expérience comme les autres. Nous y reviendrons. Mais en quoi la « brièveté de la vie » s'oppose-t-elle à leur connaissance ? Peut-être est-ce là une allusion au problème d'une justice immanente, censée être garantie par les dieux, dont on a vu qu'il était devenu un point litigieux dans la tragédie, contribuant à nourrir un refus des croyances traditionnelles. À supposer en effet qu'une forme de justice se manifeste dans l'histoire, qu'elle soit celle des cités, des lignées, ou d'une vie singulière, il faudrait, pour l'établir, accumuler des données dont l'ampleur dépasse la capacité d'enregistrement de chacun. Et l'éclatement de l'histoire en autant d'histoires qu'il y a de sujets possibles (individuels ou collectifs) rend vaine la recherche d'une réponse déterminée à la question posée.

Nous voici renvoyés au relativisme protagoréen. La proposition de « l'homme mesure » souligne la dépendance de la manifestation empirique des choses à la constitution de celui qui les appréhende : à sujets différents, objets différents, et telles les choses apparaissent à quelqu'un, telles elles sont pour lui. Impossible d'en appeler (et d'accéder) à une réalité invariable et en soi, qui serait au fondement des phénomènes. Cette thèse possède aussi une dimension sociologique (voir Théétète, 167c) : tels les valeurs, les lois et même le langage apparaissent à une communautée ${ }^{38}$, tels ils sont pour elle. Mais quel que soit le côté vers lequel on fasse pencher la proposition, vers un relativisme individuel ou collectif, dans les deux cas, c'est aussi de ce qui n'est pas que l'homme est juge : n'existe pas ce dont il ne reconnaît pas l'existence, soit parce qu'il n'est pas donné, d'une manière ou d'une autre, à son expérience, soit parce qu'il est donné, mais comme non-être.

Dans ces conditions, l'affirmation de son agnosticisme par laquelle

36. Sextus Empiricus, M. 7. 60. Cf. Platon, Sph. 232d-e : « S'agissant de tous les arts et pour chacun d'entre eux, la manière dont il faut contredire chaque artisan lui-même est étalée

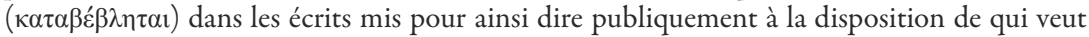
apprendre. - Il me semble que tu parles des ouvrages de Protagoras sur la lutte et les autres arts. »

37. DK80 B4 = Diogène Laërce, 9.51 ; Sextus Empiricus, M. 9.55. Cf. Platon, Théétète, $162 \mathrm{~d}$.

38. Voir Cratyle, 385e-386a. 
Protagoras ouvre son traité Sur les dieux signale en premier lieu le refus de se prononcer sur le fondement inconnaissable de ce que les hommes prennent pour des dieux. Ramenés à de simples phénomènes, ils ne sauraient faire exception au relativisme universel qu'il professe. Ne serait-il pas légitime, en effet, d'en tirer l'idée selon laquelle les dieux sont ce que les hommes croient qu'ils sont, ou ne sont pas pour autant précisément ce que les hommes croient qu'ils ne sont pas ? De sorte, après tout, que l'existence des dieux est suspendue à la croyance que les hommes leur accordent ou leur refusent ${ }^{39}$. Il est dès lors impossible à Protagoras de trancher entre convictions opposées, aussi efficientes l'une que l'autre ; il n'y aurait donc en la matière qu'une existence relative, conditionnée par le culte et la pratique des hommes, en leur diversité.

Toutefois, si Protagoras refuse de se faire la mesure de l'existence des dieux, s'il se détourne de la question, ne fraye-t-il pas alors, par sa neutralité cognitive, un chemin à l'indifférence, qui est une nouvelle façon de ne pas faire être les dieux, en les sortant du champ même du jugement humain ? Car, on s'en souvient, il y a deux manières pour l'homme d'être juge du non-être de quelque chose : la première, en ce que cette chose se donne à lui comme n'étant pas, ou comme étant fausse, irréelle, fictionnelle - ainsi en va-t-il des chimères et autres monstres mythologiques; la seconde, en ce qu'elle est hors de son domaine d'expérience et d'intérêt : elle n'existe pas pour lui, donc elle n'existe pas tout court ${ }^{40}$. C'est finalement ce qu' il advient des dieux chez Protagoras.

Son anthropologie culturelle confirme, semble-t-il, ce résultat : elle fait des dieux des créations civilisationnelles naissant d'un malentendu. En effet, le mythe que Platon met dans sa bouche - et qui, peut-on conjecturer, restitue en grande partie son enseignement - voit dans le premier culte l'expression d'une reconnaissance : les hommes rendent grâce aux dieux de ce qu'ils ont reçu. Mais en réalité, si les hommes le croient et l'imaginent, cela est sans raison ; car, des dieux au sens strict, ils n'ont rien reçu. Le thème du larcin et de la transgression prométhéenne a pour effet de libérer les hommes de toute dette. Si la ligne de partage entre les mortels et les immortels avait été respectée, l'homme aurait disparu, l'erreur d'Épiméthée lui aurait été définitivement fatale. Certes, il ne s'agit que de la première phase du mythe,

39. « En ce qui concerne les choses justes et injustes, et les choses pieuses et impies [c'est

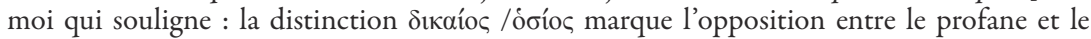
sacré, ce qui relève de la loi civile et ce qui relève de la loi religieuse], certains soutiennent avec force qu'aucune de ces choses n'étant par nature ne possède d'essence propre, mais l'opinion commune, voilà ce qui est rendu vrai, tout le temps qu'elle a pu durer et qu'elle pourra durer. » (Théétète, 172b2-6)

40. Par ce biais, on retrouve la distinction opérée par M. Martin entre un athéisme positif (avoir l'opinion que Dieu n'existe pas) et un athéisme négatif (être dépourvu d'une croyance en dieu) (Martin 2007 p. 1). 
avant l'intervention de Zeus, mais du moins le phénomène religieux tel qu'il surgit dans ces commencements de l'humanité apparaît comme un monde renversé : les hommes imaginent que leur vie est préservée par les dieux, alors que ces derniers n'y sont pour rien. De ce point de vue, la leçon du mythe rejoint en partie celle de Prodicos : les dieux résultent d'une tendance psychologique à chercher des bienfaiteurs sous les bienfaits.

En même temps, la religion dans cette première phase est encore un phénomène purement privé ; elle n'aide en rien à cimenter la cité, à renforcer le lien social, puisqu'elle précède l'aidos et la dike, et que le langage, instrument de communication, indispensable à toute mise en commun, est postérieur à son institution. Celle-ci est donc conçue comme un culte à des dieux personnels ou familiaux, certainement pas à des dieux de la cité, encore moins à des dieux universels. Le genre humain est dispersé, les cultes le sont aussi et la maxime relativiste révèle toute son efficacité : à chacun son dieu.

Néanmoins, le mythe réintroduit bel et bien in fine une religion civile : la fameuse loi de Zeus, qui punit de mort ceux qui ne sont pas capables de faire preuve d'aidos et de dike. Pourtant, cette loi n'était pas requise à première vue : si la distribution de la vergogne et de la justice a été universelle, comment peut-il se faire qu'il soit nécessaire de punir ceux qui en manquent? La réponse est triple : 1) le sens de ce qui est juste et honteux ne s'éveille qu'au contact d'un impératif, d'un « il faut » catégorique, les dispositions éthiques ne pouvant être actualisées en chacun que par la loi collective ${ }^{41}$; 2) le relativisme ne fonctionne plus à l'intérieur de la cité, laquelle ne peut laisser chacun décider de ce qui est juste ou injuste ${ }^{42}$, sauf à se déliter, et doit ainsi recourir à un fondement transcendant ; 3) la peine capitale qui en découle marque la priorité de la loi et de la vie communes sur les impératifs de survie individuelle ${ }^{43}$.

Mais il ne s'agit que des conditions générales et formelles de la vie commune, dont l'énoncé conserve l'essentiel d'un relativisme sociologique : les dieux, le contenu des lois, la détermination de l'aidos et de la dike, tout

41. Selon la logique décrite par Protagoras pour qui c'est par la délibération publique

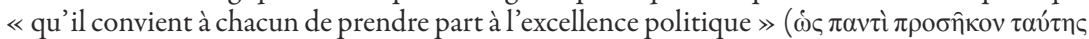

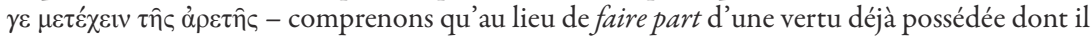
fait bénéficier la cité, il l'acquiert et la met en forme (il y « prend part ») par la prise de parole, contribuant alors au bien de la cité). Protagoras précise (323b5-7) qu'il y a un accord universel pour que chacun reconnaisse le caractère d'obligation pour lui-même des lois qui déterminent la justice, car c'est dans cette reconnaissance d'une obligation qui vient du dehors que peuvent mûrir les capacités d'éprouver la honte et d'exercer le sens de la justice.

42. « La cité les contraint à apprendre ses lois et à vivre en se conformant à leur modèle » (326d).

43. La vie selon la politique est supérieure à une vie selon la seule sophia « technique » (321d5). Celui qui vit selon cette seule dimension est le « fléau » de la cité (322d5), la

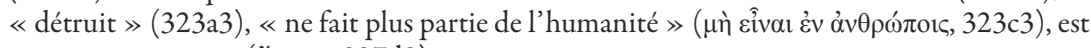
un vrai « sauvage » (ó $\gamma \rho$ ioc, 327d 3$)$. 
cela est laissé à la convention. Protagoras n'exclurait même pas, sans doute, que dans certaines circonstances, ces conditions générales ne soient pas respectées; auquel cas, la cité retomberait dans l'anomie et la violence dont «Zeus » a voulu la relever. Il appartiendrait alors à un sophiste éclairé de changer ses dispositions (cf. Théétète, 167c).

Ajoutons, avant d'examiner ce dernier point, un trait singulier du mythe de Protagoras concernant la loi de Zeus. Ce mythe, comme la plupart des mythes étiologiques, est formé de deux composantes : d'une part, un constat, le relevé d'un état de fait, ou réputé tel, susceptible à ce titre d'être discuté parce que sa pertinence outrepasse les limites du mythe et se rapporte au monde réel; d'autre part, l'« histoire », présentant comment cet état de fait est advenu, fiction qui ne trompe personne et que nul ne s'aviserait de remettre en cause, pas plus qu'on ne critique un roman en disant qu'il est inventé - il s'agit ici de l'élément proprement poïétique du mythe. D'un côté, pour emprunter le vocabulaire de la philosophie des sciences, l'explicandum (le « donné »), de l'autre l'explicans (la « cause » révélée par le mythe). Font partie de l'explicandum du mythe de Protagoras : l'habileté technique des hommes, leur aptitude à éprouver l'aidos et à sentir les exigences de la dike, une tendance à se faire la guerre, le relatif manque de spécialisation des organes corporels pour s'ajuster spontanément à un milieu déterminé. Font partie de l'explicans : la bévue d'Épiméthée, le vol du feu par Prométhée, le don par Zeus des dispositions éthiques et morales, etc. La narration insère des éléments d'une situation (l'explicandum) dans une succession d'actions accomplies par des agents dotés d'intentionnalité, mais non assignables dans l'expérience (explicans).

Nous n'avons pas de mal à répartir les éléments du mythe dans les deux premières catégories distinguées. Il y a pourtant une exception à cette dichotomie : où situer en effet la loi de Zeus ? Elle appartient tant à l'explicandum qu'à l'explicans.

Elle est incontestablement un élément de la situation des hommes dont il faut rendre compte : ils ont des lois qu'ils pensent leur venir des dieux, au même titre qu'ils sont techniciens et capables d'aidos, à la différence toutefois qu'il ne fait pas partie de l'explicandum que les hommes pensent que leurs techniques, ou l'aidos et la dike, leur viennent des dieux. Cet « état de chose $\gg$, pris en charge par le mythe, est, somme toute, exact : à observer le fonctionnement des cités, on ne peut manquer de voir qu'elles se réfèrent à des lois estampillées « loi(s) de Zeus »; toutes le font à des degrés divers ${ }^{44}$, alors que toutes n'entretiennent pas la croyance que la honte est un don divin.

Maintenant, comment le mythe en « rend-il compte »? Quel est l'explicans correspondant à l'explicandum « loi de Zeus »? La narration, ici, se

44. Cf. le préambule fameux : « L'Assemblée Nationale reconnaît et déclare, en présence et sous les auspices de l'Être suprême, les droits suivants de l'Homme et du Citoyen. » 
contente de la simple réplication de l'explicandum, muté de la sorte en explicans : nous apprenons au moment conclusif du récit que Zeus transmet par Hermès une loi dont le principal article porté à la connaissance des hommes est qu'elle est d'origine divine.

Que résulte-t-il de cette situation étrange ? Les hommes ont une loi ; c'est aussi un fait qu'ils croient qu'elle vient de dieu. Mais qu'elle vienne de dieu, c'est là une fiction, voilà la leçon du mythe. Comprenons bien : Protagoras ne fait pas de la technique, de la honte, de la justice ou de la loi de Zeus, une fiction, car ils relèvent en effet, dans la structure du mythe, du domaine de l'explicandum. Mais il fait de l'objet même de la croyance en l'origine divine de la loi une fiction, au même titre que Prométhée, Épiméthée, Zeus, puisqu'il relève de l'explicans, de la narration censée expliquer comment les choses en sont venues là. Le mythe nous dit donc que la provenance divine de la loi est à la fois une donnée comme croyance répandue et une invention comme contenu de la croyance.

Invention utile, voire nécessaire. Et, selon les circonstances, susceptible d'être modifiée et améliorée. Il s'agira alors d'une représentation « meilleure », nullement d'une représentation «plus vraie » (Théétète, 167b).

C'est exactement ce qu'entreprend un de ces « savants » dont Protagoras était tout disposé à reconnaître l'éminente valeur : Critias. Dans le fragment d'une tragédie conservé par Sextus Empiricus $(M .9 .54)^{45}$, il déploie une théorie de la genèse des dieux comme artefacts humains, privés de toute existence propre, en dehors des mythes et des fictions qui relatent leurs actions ${ }^{46}$. L'entreprise généalogique conduit naturellement à remplacer le critère de la vérité par celui de l'utilité, comme le faisait Protagoras dans le Théétète. C'est pourquoi cette perspective sophistique ne vise nullement, sur un plan pratique, à renverser les idoles, mais bien au contraire à en consolider l'usage, en mettant en lumière leur fonction véritable ${ }^{47}$.

Il fut un temps où les hommes vivaient sans ordre,

Bestialement, au service de la force brutale,

Où nul prix n'échoyait aux bons,

Où nul châtiment non plus ne frappait les méchants.

45. Certains vers de ce passage sont également cités par Aetius, qui les attribue à Euripide et soutient qu'ils dévoilent le fond de sa pensée. La paternité du texte est une question controversée et indécidable. Le nom et le genre même de la pièce sont problématiques. Pour une recension des arguments, voir Davies 1989 et Bremmer 2007. Sedley 2013 suggère un anonymat délibéré (p. 337). Whitmarsh 2014 souligne l'intérêt de la forme dramatique pour explorer à couvert des thèses radicales. Par convention, je continuerai à désigner le fragment comme étant de Critias .

46. Whitmarsh 2014 a montré que le fragment du Sisyphe reprend la forme narrative et le contenu de la Théogonie d'Hésiode et fait même allusion aux procédés du théâtre. Il en va de la fabrique du dieu comme de celle des œuvres poétiques.

47. Voir en ce sens O’Sullivan 2011-2012. 
Ensuite selon moi, les hommes établirent des lois pour punir afin que la justice règne en tyran,

$<$ Également pour tous>, et tienne asservie la démesure :

Qui commettait une faute recevait châtiment

Puis comme les lois empêchaient les hommes

De commettre au grand jour leurs actes de violence,

Mais qu' ils agissaient en secret, c'est alors, je le pense,

Que pour la première fois, un homme à l'intelligence ferme et subtile ( $\pi v \kappa v o ́ \varsigma$

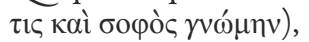

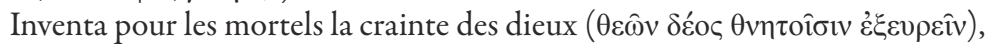
Afin d'effrayer les méchants, même lorsqu' ils dissimulent

Leurs actions, leurs paroles ou leurs pensées.

Voilà donc pourquoi il introduisit le divin (

Affirmant qu'il existe un démon $\left(\delta \alpha{ }^{\prime} \mu \omega v\right)$ florissant d'une vie impérissable,

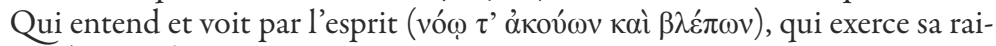

son (

Qui surveille ces choses et porte avec lui une nature divine ( рорติv),

Pour qu'il entende tout ce qui se dit chez les mortels,

Pour que voir tout ce qui s'y fait soit en son pouvoir.

Si tu viens à projeter quelque méfait, même secret,

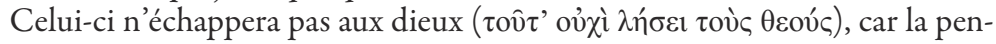
sée

Est en eux. En tenant de tels discours,

Il introduisait la plus agréable des doctrines,

Voilant la vérité sous un discours trompeur.

Il affirmait que les dieux habitent là-haut (vaícıv $\delta$ ' '̣̌ $\varphi$

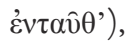

Dans le lieu dont l'évocation serait la plus terrifiante pour les hommes,

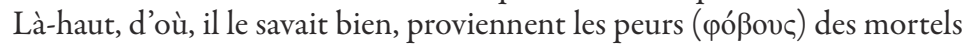

Et les fardeaux de leur vie affligée,

La voûte céleste animée d'un mouvement circulaire ( $\tau \hat{\Upsilon} \varsigma$ v̈ $\pi \varepsilon \rho \theta \varepsilon \pi \varepsilon \rho \iota \varphi \rho \rho \hat{\varsigma} \varsigma$ ),

où il voyait se produire les éclairs

Et les grondements terrifiants du tonnerre,

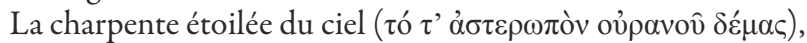

La belle ornementation produite par le Temps, sage charpentier (Xpóvov

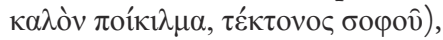

Où s'avance, brillante, la masse incandescente du soleil,

D'où la pluie humide commence son voyage vers la terre.

Il encercla les hommes de ce mur de peurs (

Et s'appuyant sur elles, par son discours, il installe de belle manière

Le démon ( tòv $\delta \alpha i ́ \mu o v)$, en un lieu qui convient,

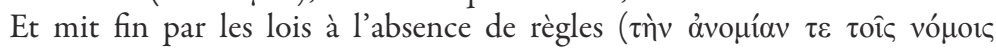
$\kappa \alpha \tau \varepsilon ́ \sigma \beta \varepsilon \sigma \varepsilon v)$.

et poursuivant un peu plus loin

C'est ainsi, je pense, que le premier quelqu'un persuada 
Les mortels de croire qu'il existe une race de démons ( $\theta v \eta \tau o v ̀ \varsigma ~ v o \mu i ́ \zeta \varepsilon v v$

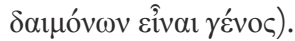

Les parallèles sont nombreux entre ce récit et le mythe prométhéen de Protagoras dans le dialogue de même nom. Même situation initiale ${ }^{48}$ : l'allusion à la vie de « bête sauvage » dans les temps qui précédèrent l'institution de la loi trouve son parallèle dans la destruction de l'humanité par les bêtes sauvages et l'hostilité mutuelle des hommes ${ }^{49}$ avant la distribution de l'aidos et de la dike. Un remède également déployé en deux temps : l'art démiurgique d'abord, l'art politique ensuite, pour le mythe de Protagoras, la loi d'abord, la justice divine ensuite, pour l'histoire contée par Critias. Mais l'art politique du premier prend son appui normatif sur la loi de Zeus, retrouvant ainsi le dieu pourvoyeur de justice du second.

Les différences sont également claires. Il n'est nullement question, chez Critias, de don du feu ni de dispositions à la vertu universellement distribuées. Surtout, le cadre mythique est éliminé, même si le fragment est issu d'une pièce comprenant des éléments mythiques : dans le passage concerné, les dieux ne sont pas les acteurs de l'histoire, ils sont en revanche l'objet de croyances organisées à des fins politiques déterminées. Ce point, il est vrai, était déjà suggéré dans le récit de Protagoras par le truchement paradoxal du mythe, sans être cependant abordé par la voie plus directe, mais plus périlleuse, du logos proprement dit. Voie dans laquelle, pour sa part, Critias s'engage plus résolument et plus clairement, bien que ce soit le personnage qui s'exprime ici et non l'auteur en personne. Il a su également mobiliser toute une série de topoi caractéristiques des diverses écoles de pensée de son temps pour les intégrer à son projet généalogique.

Le dieu dont Critias restitue la fabrication, c'est celui que déjà Xénophane avait théorisé, en voie vers une forme de monothéisme, un dieu seul en son genre qui « voit, entend et saisit par toutes les parties de son être » $(21 \mathrm{DK}$ A1. 19, B24) et qui perçoit également tout, puisque « sans aucune peine, il fait tout (panta) trembler par la faculté de son intelligence (noou phreni) » $(\mathrm{B} 25)^{50}$. Tout ce qu'affecte son intelligence lui est nécessairement connu. Critias conserve donc de Xénophane l'idée d'une connaissance divine résidant essentiellement dans une perception panoptique ${ }^{51}$.

48. Comme le fait observer Davies 1989, p. 18, les deux récits commencent par la même phrase.

49. Pour le même assemblage de termes, voir Diodore de Sicile, 1.8, dont la source est peut-être Démocrite (68DK B5.1).

50. Simplicius introduit la citation constitutive du frag. 25 de la manière suivante : « et affirme-t-il (Xénophane), il (le dieu) pense toutes les choses (panta), quand il (Xénophane) $\operatorname{dit}:[\ldots] \gg$.

51. Aristote lie le dieu de Xénophane au ciel, comme le fait à sa manière Critias pour son dieu : « considérant le ciel entier, il (Xénophane) déclare que l'un est dieu, ou le dieu est un » (Métaphysique, 986b18-27). 
L'autre leçon tirée de Xénophane est celle de la projection anthropomorphique. Xénophane avait bien vu que la représentation des dieux était une sorte d'extension de la représentation que les hommes pouvaient se faire d'eux-mêmes. Toutefois, Xénophane épargnait de sa critique la dimension cognitive et directive du dieu, qui lui semblait relever de sa véritable nature (il écoute, il voit, il use de son intelligence, il dirige). Or Critias considère pour sa part que l'ensemble de ces caractéristiques résulte du même anthropomorphisme qui fait que les dieux des Éthiopiens sont noirs et au nez camus et que si les bœufs avaient su dessiner, ils auraient donné aux dieux une apparence bovine. Dans tous les cas, nous avons affaire à une construction par projection sur un support fictif de qualités humaines (ou animales) portées à leur plus haut degré de pénétration et de puissance.

Critias souligne d'ailleurs la proximité entre d'une part les caractéristiques de l'homme avisé, qui s'efforce de remédier à la faiblesse des lois, et d'autre part le personnage qu'il fabrique au terme de ses recherches; le premier est doté d'une « intelligence ferme et subtile », tandis que le dieu qu'il invente est lui-même «phronon ». Et la capacité de perception de ce dernier est supposée prolonger et pallier celles, défaillantes, des mortels, incapables de se surveiller les uns les autres de nuit comme de jour.

Dans l'Antiquité, certains étaient conscients de cet artifice par lequel les hommes pénétrants construisent une sorte de double agrandi d'eux-mêmes qu'ils mettent au premier rang de l'univers. Démocrite, dans le fragment 30, évoque ces « doctes » (logioi) qui lèvent leurs mains

vers ce que nous, Hellènes, nous appelons l'air, en disant : « Sur tout, Zeus délibère en lui-même, il sait, donne et retire tout et il règne sur tout. »

Faut-il voir dans ce geste et dans cette déclaration les actes d'institution du dieu ? Toujours est-il que Démocrite reconnaît la fonction politique de cette supposée perception divine :

que l'on croie que les dieux voient tout, et l'on ne commettra pas de faute ni en cachette ni en public. (B112)

Platon signalera avec ironie, pour sa part, combien les « sages » s'enorgueillissent ${ }^{52}$ en imaginant un dieu intelligent gouvernant le cosmos (avant lui-même d'aller dans leur sens) :

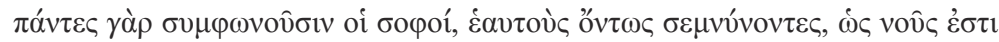

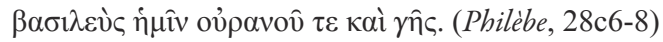

Cette «symphonie » des « sages » réunit, par-delà leurs différences, Xénophane, Anaxagore, Démocrite, Alcméon et ... Critias.

52. Voir un même biais « scolastique » chez la grue dans le Politique, qui « s'enorgueillit » (263d7) semblablement en croyant que l'opposition intelligence / non-intelligence suffit à la mettre à part comme espèce. Cet orgueil n'est jamais pris en bonne part par Platon (Théétète, 175a6; Phèdre, 243a1). 
De fait, en situant son dieu dans le ciel, Critias pouvait récupérer toute la religion astrale qui avait les faveurs de quelques penseurs de l'époque. Certes, tous n'acceptaient pas de loger dans le soleil ou dans les étoiles l'intelligence qui gouverne le monde, et certains (Anaxagore et ses disciples) adoptaient à l'égard des planètes un matérialisme assez cru, qui parut à beaucoup choquant. Critias n'écarte pas pour autant le secours qu'ils pouvaient apporter à sa cause (du fait de l'intelligence qu'ils maintenaient dans l'univers). Ainsi, sa description du soleil comme «masse incandescente » est celle-là même d'Anaxagore ${ }^{53}$. Toutefois, en inscrivant le mouvement de l'astre du jour dans celui du ciel tout entier, en le rattachant à l'œuvre du temps et à la pluie qui féconde la terre, Critias fait également écho à cette tradition divinisant le soleil et ses bienfaits que rapporte Prodicos ${ }^{54}$ et dont on trouve des traces chez Sophocle ${ }^{55}$. Le début de l'Agamemnon d'Eschyle (4-6) mentionne « l'assemblée des astres nocturnes et les princes éclatants

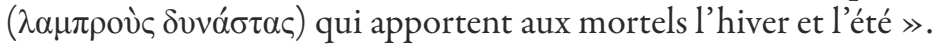

En outre, Critias met l'accent sur l'ordre qui se manifeste dans le ciel (contrastant avec l'état ó $\tau \alpha \kappa \tau o \varsigma$ de l'humanité primitive), en évoquant « la voûte céleste animée d'un mouvement circulaire » ( $\tau \hat{\uparrow} \varsigma$ Ü $\pi \varepsilon \rho \theta \varepsilon \pi \varepsilon \rho 1 \varphi \circ \rho \hat{\alpha} \varsigma)$, lequel fait écho à la révolution de la lune qui figure dans le programme de recherche qu'Aristophane met au compte de Socrate (Nuées, 172, voir a contrario Xénophon, Mémorables, 4.7.5) et au mouvement du ciel dans son

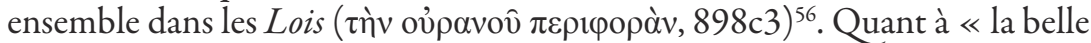

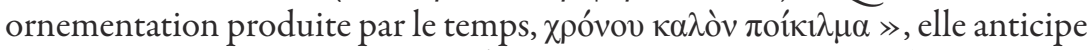

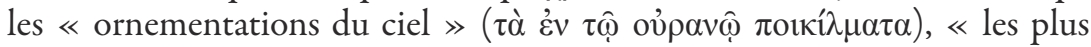
belles et les plus précieuses » (Platon, $R$. VII, 529c7-d1).

Et si le temps est ce « sage charpentier », sans doute est-ce d'abord en raison de la régularité des révolutions astrales et des cycles cosmiques qui se répètent avec exactitude, attestant l'intelligence présidant aux mouvements des cieux qui scandent le devenir. On pourrait, à nouveau, y voir la préfiguration du thème platonicien du «temps, image mobile de l'éternité » façonnée par le démiurge, mais c'est d'abord un emprunt au poète comique Cratès, qui soulignait par la même formule l'alternance régulière de la mort et de la vie :

Le temps ne cesse de faire retour pour moi : sage charpentier ( $\tau \varepsilon \dot{\kappa} \tau \omega \nu \mu \grave{\varepsilon} v$ бофóc), il œuvre à rendre toutes les choses plus faibles, mais à nouveau

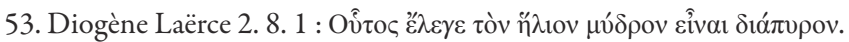

54. Déification du soleil : 84DK B5 (= Sextus Empiricus, M. 9.18 ; 9.52). Cf. « Zeus qui fait pleuvoir », 84DK B5 (= Thémistius, Or. 30.349a-b).

55. Fr. 752, Radt 1977 : il connaît des « sages (бọoí) qui appellent le soleil le géniteur des

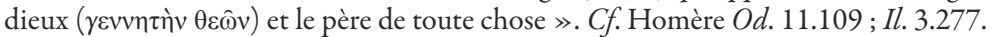

56. Proximité aussi avec Alcméon de Crotone, qui le premier a posé le principe d'une âme automotrice (24DK A1, A12). Soleil, lune et étoiles se meuvent chez lui d'un mouvement circulaire - qui peut prendre des directions différentes (A4). C'est la perfection de ce mouvement qui justifie leur divinité. 
fleurissent celles qu'il nourrit, comme le jeune bétail ou les jeunes baies. (39.1-40.1 Kock 1880)

À cet ordre cosmique est associé un ordre providentiel, deuxième aspect de la sagesse et de l'industrie du temps, comme le suggère un vers parallèle chez Euripide ${ }^{57}$, prononcé par le chœur dans le Bellérophon, sans doute dans une réplique à l'impiété du personnage principal : « le temps, qui n'est fils de personne, applique les règles de la justice et révèle dans tous les cas les forfaits humains $\gg$ (fr. 303 Nauck 1912) ${ }^{58}$.

Ce faisant, Critias ne procède pas à un décalque direct des doctrines théologiques de ses prédécesseurs ou contemporains, puisqu'il ne les prend pas au premier degré ; mais il se sert de ces discours pour montrer comment construire un dieu rationnel pilotant le cosmos, le passage de l'intelligence humaine à l'intelligence divine s'accompagnant d'un alignement de leurs manifestations respectives : l'ordre politique fragile et instable de la première s'ajuste sur l'ordre cosmique majestueux et durable de la seconde.

Mais une troisième élaboration intègre, selon Critias, les deux premières (cosmologique et providentielle) et les subsume : dans ce qui fait peur (les éclairs, la foudre, le tonnerre), l'homme avisé enseigne à déceler de manière systématique une intention punitive, à sentir la présence d'une menace, à reconnaître la perspective d'une sanction. Sans doute convient-il de cultiver, à la façon de Prodicos, le sentiment de gratitude face aux avantages et aux bonheurs de la vie. Mais il importe surtout de développer le sens d'une responsabilité personnelle et collective vis-à-vis des « colères » de la nature, qui vengent un certain ordre dérangé par la conduite des hommes qui violent le nomos. Ici la source semble être démocritéenne : le philosophe d'Abdère soutenait que les dieux seraient nés de l'étonnement et de la terreur des hommes (A75) face à des événements météorologiques impressionnants ${ }^{59}$.

Ces trois projections contribuent à définir une seule et même puissance divine, dont l'unité est rendue sensible par son lieu de résidence (le ciel) et dont la grandeur et l'autorité seraient attestées par l'ampleur et la diversité des phénomènes qui en portent la marque. Au final, c'est la peur du divin

57. À ma connaissance, cet élément est passé inaperçu dans la querelle en paternité à propos du fragment du Sisyphe.

58. Pour une convergence plus explicite (et postérieure) entre astronomie et police, voir Plaute qui, dans le prologue du Rubens, fait parler une étoile, l'Arcture, envoyée par Jupiter pour surveiller et sanctionner les hommes, notamment ceux qui ne tiennent pas parole. Diodore de Sicile rapporte que les Chaldéens associent également des dieux à des astres, appelés « dieux conseillers » ou encore « juges de l'univers », dont la fonction est d' « inspecter tout ce qui se passe parmi les hommes et dans le ciel » (2.30).

59. Démocrite aurait également soutenu qu'ils ne sont rien d'autre que des simulacres (68DK A74, 78), émanations des phénomènes naturels qui frappent inconsciemment l'être humain (A74, 78, B166), et que ce dernier réifie au moyen de conventions nominales (B142). Les dieux n'auraient aucune existence en dehors de ces images (B166, voir aussi B129). 
qui parachève la vertu chez les hommes ${ }^{60}$. Si le commencement de celle-ci découle d'un intérêt bien compris et d'une forme rudimentaire de contrat, son perfectionnement dépend d'une autorité transcendante, grâce à laquelle chacun tient son rôle, même quand il se dérobe au contrôle des pairs ${ }^{61}$. C'est la manière dont les hommes et les sociétés ont résolu, selon Critias, le problème du passager clandestin tel que le posera le mythe de Gygès dans la République.

\section{Les Lois de Platon : de l'athéisme à la religion d'État}

Platon hérite de cette pensée sophistique dont nous avons recensé quelques-unes des figures. Il dresse, comme on sait, un panorama de l'impiété au livre $\mathrm{X}$ des Lois, dont l'une des trois formes correspond à un athéisme strict, la négation de l'existence des dieux, les deux autres renvoyant à une mécompréhension des relations entre hommes et dieux.

Je voudrais montrer que la réponse de Platon aux athées leur doit beaucoup : elle est en particulier fort proche de celle de Critias, sans qu'il le confesse, naturellement. En effet, l'enjeu pédagogique des Lois est de régler les dispositions et les croyances : reconnaître d'abord celles dont les effets sont délétères pour la communauté politique, les transformer ensuite pour prévenir de tels effets. L'approche est fonctionnaliste avant d'être strictement philosophique. À cet égard, le rôle instituant du nomos et du législateur est constamment souligné par l'Athénien.

Jamais homme que les lois ont persuadé de l'existence des dieux n'a de plein gré commis un acte impie, ou proféré une parole criminelle ; il n'a pu le faire qu'induit par l'une des trois déclarations suivantes, soit comme je l'ai dit, qu' ils n'existent pas, soit en second lieu qu' ils existent, mais n'ont aucun souci des humains, soit enfin qu'ils sont faciles à fléchir et se laissent retourner par des prières et des sacrifices. (885b4-9)

Il y a toujours eu, plus ou moins, des esprits atteints de cette « maladie » - entendons le strict athéisme (888b7-8). Mais c'est une maladie de jeunesse; nul ne persiste en cette opinion, selon l'Athénien : avec l'âge (et la mort

60. Riedweg conjecture que le terme manquant du dernier vers du fragment $286 \mathrm{du}$

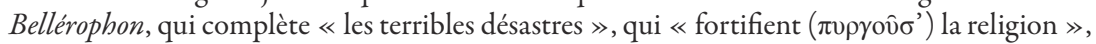

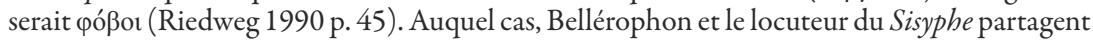
la même conviction à propos de l'origine de la religion, mais dans un cas, ce serait sous la forme de la dénonciation, dans l'autre, sous la forme d'une approbation. Autre pièce à verser au dossier en paternité.

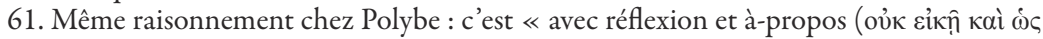

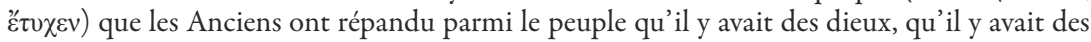

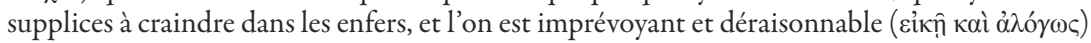
dans notre siècle de rejeter ces sentiments » (VI, 56, 12). 
approchant), il y renonce $(c 4)^{62}$.

Un facteur culturel et conjoncturel a pu toutefois accroître la diffusion de la maladie et contrecarrer ce qu'enseignent les lois. Les croyances impies peuvent en effet refléter, toujours selon l'Athénien, une ignorance très fâcheuse, répandue par deux sortes de discours, celui des anciens, les poètes mythologistes, qui décrivent la naissance des dieux, leurs faits et leurs méfaits, et celui des modernes, les naturalistes. Les premiers, auteurs antiques et vénérables, n'offrent pas de modèle satisfaisant du respect dû aux parents (allusion aux théogonies violentes d'Hésiode). Les seconds sont des matérialistes pour lesquels les dieux astraux ne sont que pierre et terre (les « Fils de la terre » du Sophiste n'admettaient comme être que ce qu' ils peuvent toucher et saisir de leur main ; et si l'on songe à Anaxagore, son dieu cosmique, distinct des astres, était étranger à la préoccupation du meilleur).

L'ignorance de ces athées passe pourtant « pour la plus grande sagesse »

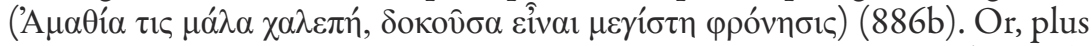
tôt dans les Lois, il était montré que cette amathia, la plus grande (689d5), ne devait pas se caractériser «logiquement » par une certaine paresse de pensée ou un défaut de raisonnement (689c9-d1), mais bien plutôt, « éthiquement », par l'absence du cadre moral à l'intérieur duquel l'argumentation doit se déployer pour être pertinente. La conduite peut d'ailleurs rester sensée même quand elle ne s'accompagne pas du logos adéquat (689d4), car le cadre suffit pour procurer l'harmonie, la sumphonia, nécessaire (d7). Dans les Lois, la vertu des citoyens ne repose pas d'abord sur la connaissance, ni sur l'opinion droite, mais sur des formes de dispositions à croire, à sentir et à agir : le vertueux est un être bien dressé par des habitudes appropriées (ỏ $\theta \hat{\omega} \varsigma$

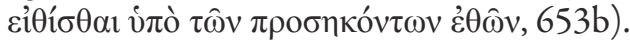

C'est pourquoi l'Athénien regrette la simplicité des premiers temps, où les hommes n'auraient jamais pu soupçonner, faute de la sophia des modernes, un mensonge dans l'enseignement sur les dieux et sur la vie humaine qu'ils recevaient de la cité et auquel ils adhéraient en toute confiance (679c7).

Quant aux contemporains, il est difficile de les arracher par le seul raisonnement à un point de vue gouverné par un moi injuste, privilégiant le plaisir présent : les gains et les peines futurs sont obscurcis. Pour corriger cet effet de perspective, il faudrait au législateur, selon l'Athénien (qui suit ici exactement la démarche décrite par Critias), un mensonge, « le plus utile des mensonges » (663e), celui qui produira pour la cité le plus grand bien, la capacité de parler d'une seule voix en la matière (664a).

Or cette unité d'expression dans les chants, les mythes et les discours (sur le bonheur de la vie juste), trouve sa note fondamentale dans le discours du législateur (663b). Le vaste préambule du livre $\mathrm{X}$ enseigne cette providence

62. Cf. Eschyle dans les Perses (497-498) : ceux qui niaient les dieux se mettent à les implorer à l'approche de la mort. Sentiment semblable en République (330e1). 
qui garantit au juste la vie la plus heureuse et à l'injuste la vie la plus malheureuse. Démonstration qu'il est « pénible » de devoir administrer, mais qui est d'autant plus requise qu'elle s'adresse à des esprits insuffisamment conditionnés par les rites et les mythes. L'ambition est de faire naittre en leur âme, précisément comme le voulait Critias, une crainte salutaire $(887 \mathrm{a} 6-7)^{63}$.

La théologie du livre $\mathrm{X}$, qui préface l'ensemble du corpus législatif des $L$ ois $(887 \mathrm{c} 1)$, possède elle-même une valeur légale. Devenue intangible, elle ne souffrira pas des infirmités de l'écrit. L'immobilité, la réitération des mêmes propos, l'absence d'un père du discours que l'on pourrait interroger à loisir, tous ces éléments qui induisaient le procès platonicien d'une écriture paralysant la pensée, se trouvent à présent réinvestis positivement d'un tout autre point de vue : ils facilitent la mémorisation, ils s'accordent à l'uniformité de conviction, ils façonnent autant qu'ils expriment le caractère collectif de la croyance ; autant d'avantages, donc, pour la mise en conformité des esprits.

À l'autre bout de cette préface théologique et pédagogique, au terme de la réfutation de l'impiété, la religion officielle voit son monopole idéologique et pratique conforté par l'interdiction de tout culte privé. Une série de sanctions sont également prévues à l'encontre des athées qui persévéreraient dans leurs erreurs pernicieuses.

L'Athénien distingue deux espèces principales de châtiment. La première frappe les licencieux, les magiciens ou les prêtres qui se flattent d'un commerce spécial avec les dieux pour obtenir d'eux certaines faveurs particulières, ou encore les individus habiles prétextant un relativisme des croyances pour se faire les défenseurs du droit naturel du plus fort : ils subiront une double peine, l'exil social, d'abord, puisqu'ils seront enfermés dans une prison au centre du territoire, dans un lieu « désert et sauvage » (à l'image de leur croyance en un monde « vide de dieux »), la mort symbolique ensuite, puisque leur dépouille sera jetée hors des frontières du territoire national, sans sépulture. Quant aux athées vertueux, dotés d'un caractère juste, ils seront cinq ans durant emprisonnés aux frais de la cité, à proximité du conseil nocturne. Nul ne leur rendra visite, à part les membres dudit conseil, citoyens parmi les plus éminents de la cité que distinguent le savoir et la rectitude de la conduite, qui chercheront à imprimer en l'esprit du condamné de nouvelles dispositions, en s'entretenant avec lui du salut de l'âme (908a4-5). La scène discrètement suggérée par Platon peut faire songer aux entretiens de Socrate avec ses disciples, alors qu'il est emprisonné pour crime d'impiétéét.

63. $C f$. Isocrate à propos des Égyptiens : « ceux qui font paraître les faveurs des dieux et leurs punitions plus grandes qu'elles ne le sont en réalité ont rendu un grand service à la vie des hommes $\gg$ (Busiris, 24).

64. Mêmes lieux (prisons), mêmes temporalités (précédant l'exécution), mêmes contextes (accusation d'athéisme), mêmes interrogations (sur le salut de l'âme). Des différences bien sûr : dans les Lois, l'entretien est secret et les interlocuteurs sont dans une relation asymétrique 
Elle évoque davantage, à la faveur d'un anachronisme assumé, l'entretien du Christ avec le grand inquisiteur dans les Frères Karamazov : il s'agira en effet, dans le cadre de ces rencontres clandestines, de convaincre l'athée de l'utilité de cette croyance aux dieux pour le salut d'une âme qui est moins, sans doute, celle de la personne elle-même que celle de la cité tout entière, en tout cas de ses membres. S'il est finalement exécuté, c'est parce que sa fidélité à ses convictions le rend dangereux à long terme pour la cité, tout comme l'acosmisme du Christ le condamne à la mort dans l'intérêt même de la collectivité, selon le grand inquisiteur.

Le contexte dans lequel s'inscrit la réfutation des athées est donc clair : la religion telle qu'elle est conçue et défendue au livre $\mathrm{X}$ est essentiellement politique. Sous le contrôle exclusif de la cité, elle a pour mission d'assurer la conviction que l'ordre de la cité s'insère dans un ordre du monde, qu'il y a une forme de continuité de l'un à l'autre, et donc une forme de justice immanente où ceux qui dérogent aux ordres de la raison et du nomos seront durement sanctionnés dans le cours de leurs existences multiples.

Cette continuité conserve le caractère traditionnel de la justice divine, tout en le renouvelant profondément. D'une part, les dieux n'interviennent plus directement, ne prononcent pas de jugement ad hoc. Un nouveau parallèle s'esquisse avec le discours de Critias, puisque lui non plus ne faisait pas état d'un jugement après la mort. La fonction des dieux se résume dans les Lois à la mise en ordre et à la conservation de cet ordre dans l'univers, et cela suffit à assurer en principe la justice ${ }^{65}$ : une quasi-physique des passions (903e-904b) conduit naturellement celui qui se livre aux désordres de l'âme à en souffrir de plus en plus, sans pouvoir même espérer que la mort l'en soulage.

D'autre part, les dieux ne prescrivent rien aux hommes : pas de commandement ou d'impératif qui découlerait de la nature ou de la volonté divine.

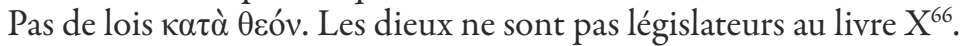

En revanche, les dieux sont des éléments internes à la législation. On se souvient qu'aucune conduite répréhensible ne pouvait être imputée, selon l'Athénien, à ceux qui avaient été persuadés par les lois de leur existence (885b4). La réfutation proprement dite doit permettre aux législateurs de se défendre contre l'accusation d'avoir fait « quelque chose d'épouvantable, en instituant la croyance aux dieux par la loi » (887a1).

C'est une constante dans tout le passage : les dieux auxquels il est fait référence, ceux qu'il importe de justifier, ce ne sont pas les dieux en eux-mêmes,

de pouvoir (de vie et de mort), ce qui n'est pas le cas dans le Criton et le Phédon. Et, bien sûr, Socrate et l'athée des Lois défendent des thèses opposées à propos des dieux.

65. Voir Saunders 1973.

66. L'Athénien joue ailleurs avec l'idée de faire accroire l'origine divine des lois (par ex. 634e; 657a-b). 
ou par nature, mais bien les dieux tels qu'ils ont été institués par les lois. À l'orée de l'apologia pro vita deorum, le jeune destinataire de la réfutation est prévenu :

il revient à celui qui fait les lois de t'apprendre maintenant comme plus tard

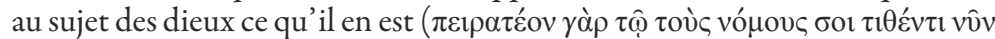

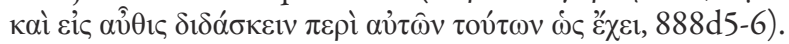

Et dans le cours de la démonstration, il est rappelé qu'il s'agit, à l'encontre des athées, de défendre les dieux

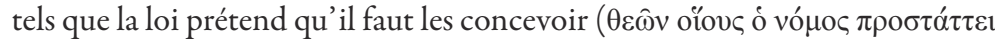

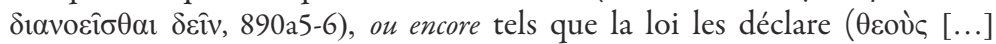

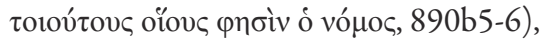

quitte, comme l'avoue Clinias, à devoir sortir des sentiers battus pour justifier

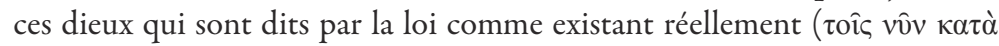

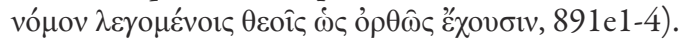

Et, au terme de la démonstration, ce sont bien « les dieux qui existent selon

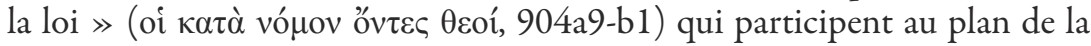
providence divine, à laquelle les hommes doivent croire, en se montrant « dociles à la parole du législateur » et à ce qu' « énonce la loi de nos pères

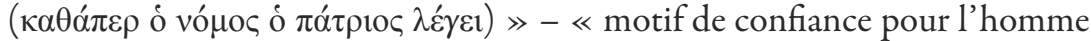
de bien, de frayeur pour le méchant $\gg(959 \mathrm{a} 4-\mathrm{b} 6)^{67}$.

Soit, dira-t-on. Mais n'y a-t-il pas néanmoins un véritable projet philosophique de démonstration de l'existence des dieux ? Incontestablement, le livre X des Lois paraît offrir une tentative de ce genre qui, avec un court passage du Philébe, constitue dans la tradition philosophique occidentale le premier essai pour prouver dieu. Nous aurions affaire à un discours authentiquement théologique, visant le vrai et non seulement ce qu'il serait utile de faire croire.

Ce n'est pas le lieu de discuter en détail l'argumentation déployée, ni d'en examiner les fragilités, notamment celles dont Platon pouvait être conscient. L'intention de ce dernier reste toutefois claire : établir un lien entre les révolutions célestes et l'organisation de la cité, mais selon une dépendance paradoxale. L'autonomie du monde fournit en effet à la cité (et à l'âme) l'injonction à sa propre autonomie et lui en donne les critères formels : cohérence, constance, précision, clôture. Mais quant au contenu, les dieux astraux ne proposent aucun modèle à imiter. La souveraineté de la loi et du législateur est, à cet égard, complètement préservée, y compris en matière religieuse. Platon élabore plus subtilement que Critias le lien entre macrocosme et microcosme, tout en conservant l'essentiel de sa leçon politique.

67. Quand l'Athénien envisage que « la cité tout entière ait à recueillir les fruits de l'impiété de quelques-uns, non sans quelque justice » (910b6), il reprend l'antique croyance d'Hésiode (voir note 34) pour le bénéfice politique que la cité est susceptible d'en retirer, non

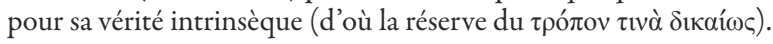




\section{Conclusion}

Loin d'être complètement marginal ou périphérique, l'athéisme a largement trouvé sa place parmi les préoccupations des penseurs de l'âge classique. Rien pourtant de militant, car il s'inscrit en creux dans la description anthropomorphique et conventionnaliste du fait religieux. Description qui ne vise pas à combattre la croyance aux dieux, mais à montrer ses ressorts et son utilité, à évoquer ce qui est susceptible de la fragiliser dans le monde humain (Euripide), ou de nature, au contraire, à en accroître l'empire et le bénéfice collectif (Protagoras et Critias). Tous partagent un même trait définitoire dans leur approche du polythéisme : les dieux sont le fruit d'une tradition, ils sont conçus et déterminés par la loi ancestrale ; mais ils contribuent inversement à son maintien et à son respect. L'aisance avec laquelle ces penseurs justifient par des considérations politiques la religion sans s' inquiéter du potentiel destructeur de cette justification tient justement à la plasticité des croyances et des cultes. Nous ne sommes pas dans le contexte d'une loi de Dieu, d'une révélation codifiant la foi et la pratique sur la base d'un texte inaltérable, procédant de la divinité elle-même. Certes, Platon a voulu donner à sa théologie la forme même d'une législation. Mais c'est d'abord pour soumettre la première à la seconde. C'est aussi pour fixer ce que sont les dieux, non pour rapporter leurs commandements. C'est enfin pour offrir un cadre rationnel à la liberté politique. Les auteurs athées du $\mathrm{V}^{\mathrm{e}}$ siècle les plus explicitement constructivistes dans leur approche du fait religieux ne désavoueraient pas un tel projet. 


\section{BIBLIOGRAPHIE}

Assmann, J. 2001 : Moïse l'Égyptien : un essai d'histoire de la mémoire, trad. de l'allemand par L. Bernardi, Paris, 2001 (Collection historique).

Blaise, F. 2003 : «L'expérience délirante de la raison divine : Les Bacchantes d'Euripide », Methodos, 3 (2003), en ligne : http://methodos.revues.org/173 (DOI : 10.4000/methodos.173).

Bremmer, J. 2007 : « Atheism in Antiquity », dans M. Martin (éd.), The Cambridge Companion to Atheism, Cambridge, 2007, p. 11-26 (Cambridge Companions to Philosophy).

BURKeRT, W. 1985 : Greek religion, transl. by J. Raffan, Cambridge (Mass.), 1985.

Burnet, J. 1924 (éd.) : Plato, Euthyphro, Apology of Socrates, Crito, Oxford, 1924.

Couloubaritsis, L. 1989 : «Réflexions de Sextus Empiricus sur les dieux (Adv. Math., IX) », Kernos, 2 (1989), p. 37-52.

Couissin, P. 1941 : « Les sorites de Carnéade contre le polythéisme », Revue des Études Grecques, 54/254, 1941, p. 43-57.

Davies, M. 1989 : « Sisyphus and the Invention of Religion ('Critias' TrGF 1 (43) $\mathrm{F} 19=\mathrm{B} 25 \mathrm{DK}) \gg$, BICS, 36/1 (1989), p. 16-32.

Dindorf, W. 1832 (éd.) : Themistius, Themistii orationes ex codice mediolanensi, Leipzig, 1832.

DodDs, E. R. 2009 : Les Grecs et leurs croyances, trad. de É. Helmer, Paris, 2009 (Les Marches du Temps).

Dorival, G. et D. Pralon, 2002 (éd.) : Nier les dieux, nier Dieu : actes du colloque organisé par le Centre Paul-Albert Février (UMR 6125) à la Maison Méditerranéenne des Sciences de l'Homme les $I^{\text {er }}$ et 2 avril 1999, Aix-en-Provence, 2002 (Textes et documents de la Méditerranée antique et médiévale, 2).

FAHR, W. 1969 : Theous nomizein : Zum Problem der Anfänge des Atheismus bei den Griechen, Olms, 1969 (Spudasmata, 26).

Febvre, L. 1942 : Le Problème de l'incroyance au XVI siècle : la religion de Rabelais, Paris, 1942 (L'Évolution de l'humanité, 53).

Foucault, M. 1966 : Les Mots et les Choses: une archéologie des sciences humaines, Paris, 1966 (Bibliothèque des sciences humaines).

Husson, S. 2014 : « Parrhèsia socratique et parrhèsia cynique : le cas de l'injure », Cahiers « Mondes anciens », 5 (2014), en ligne: https://journals.openedition. org/mondesanciens/1256 (DOI : 10.4000/mondesanciens.1256).

Kannicht, R. 2004 (éd.) : Euripide, Tragicorum graecorum fragmenta, vol. V : Euripides, Göttingen, 2004.

Kock, T. 1880 (éd.) : Crates, Comicorum atticorum fragmenta, volumen I : Antiquae comoediae fragmenta, Leipzig, 1880.

Lefkowitz, M. 2003 : « "Impiety" and "Atheism" in Euripides' Dramas », dans J. Mossman (éd.), Euripides, Oxford, 2003, p. 102-112 (Oxford Readings in Classical Studies).

Martin, M. 2007 : «General introduction » dans M. Martin (éd.), The Cambridge Companion to Atheism, Cambridge, 2007, p. 1-7 (Cambridge Companions to Philosophy). 
Narbonne, J.-M. 2016 : Antiquité critique et modernité: essai sur le rôle de la pensée critique en Occident, Paris, 2016.

Nauck, A. 1912 (éd.) : Euripide, Euripidis tragoediae, volumen III : Perditarum tragoediarum fragmenta, Leipzig, 1912 (Bibliotheca scriptorum graecorum et romanorum Teubneriana, Auctores graeci).

O'Sullivan, P. 2011-2012 : «Sophistic Ethics, Old Atheism, and "Critias" on Religion », Classical World, 105/2 (2011-2012), p. 167-185.

RADT, S. 1977 (éd.) : Sophocle, Tragicorum graecorum fragmenta, vol. IV, Sophocles, Göttigen, 1977.

Riedweg, C. 1990 : « The "Atheistic" Fragment from Euripides' Bellerophontes $\left(286 \mathrm{~N}^{2}\right) \gg$, Illinois Classical Studies, 15 (1990), p. 39-53.

Saunders, T. J. 1973 : « Penology and Eschatology in Plato's Timaeus and Laws », Classical Quarterly, 23 (1973), p. 232-244.

SCHEID, J. 2005 : Quand faire, c'est croire : les rites sacrificiels des romains, Paris, 2005 (Collection historique).

Sedley, D. 2013 : « The Atheist Underground », dans V. Harte \& M. Lane (éd.), Politeia in Greek and Roman Philosophy, Cambridge, 2013, p. 329-348.

Vernant, J.-P. 1985 : « Le Dionysos masqué des Bacchantes d'Euripide », L'Homme, 25/93 (1985), p. 31-58.

Whitmarsh, T. 2014: « Atheistic Aesthetics : The Sisyphus Fragment, Poetics and the Creativity of Drama », CCJ, 60 (2014), p. 109-126.

- 2015 : Battling the Gods : Atheism in the Ancient World, New York, 2015.

- 2016: «Diagoras, Bellerophon and the Siege of Olympus », JHS, 136 ( 2016), p. 182-186.

Winiarczyk, M. 1981 (éd.) : Diagorae Melii et Theodori Cyrenaei reliquiae, Leipzig, 1981 (Bibliotheca scriptorum graecorum et romanorum Teubneriana. Auctores graeci).

- 1984 : «Wer Galt im Altertum als Atheist? », Philologus, 128/2 (1984), p. 157-183. 